\title{
Long duration of immunity against a type 1 heterologous PRRS virus challenge in pigs immunised with a novel PRRS MLV vaccine: a randomised controlled study
}

\author{
Jeremy Kroll ${ }^{1 *}$, Mike Piontkowski ${ }^{2}$, Poul H. Rathkjen ${ }^{3}$, Francois-Xavier Orveillon ${ }^{3}$, Christian $\mathrm{Kraft}^{4}$ \\ and Oliver G. Duran ${ }^{3}$
}

\begin{abstract}
Background: Porcine reproductive and respiratory syndrome virus (PRRSV) is widespread in commercial pig farms worldwide, and has a significant cost to the swine industry. Herd owners need a vaccine that will confer long-lasting immunity to prevent PRRSV infection and transmission. The studies described here evaluated duration of immunity conferred by a European-derived PRRS (isolate 94,881) modified live virus (MLV) vaccine, Ingelvac PRRSFLEX EU, at 20, 24 , and 26 weeks post-vaccination. Primary endpoints were the assessment of gross and histological lung lesions and viral RNA load in lung tissue 10 days following heterologous PRRSV challenge. Secondary endpoints included clinical observations, average daily weight gain (ADWG) and viral RNA load in serum 10 days post-challenge. Three blinded, vaccination-challenge efficacy studies were performed using separate cohorts of pigs ( $n=56$ per study). Pigs received either Ingelvac PRRSFLEX EU (Group 1) or placebo (Groups 2 and 3). Groups 1 and 2 were subsequently challenged with heterologous European PRRSV isolate 205,817 at 20, 24 or 26 weeks post-vaccination.
\end{abstract}

Results: Mean gross lung lesion scores were significantly lower in Group 1 than in Group 2 at 24 and 26 weeks $(p<0.0001)$, but not at 20 weeks $(p=0.299)$. Significantly lower mean histological lung lesion scores were observed in Group 1 versus Group 2 at 20 ( $p=0.0065), 24(p<0.0001)$ and 26 weeks $(p<0.0001)$. Mean viral RNA load in lung tissue was significantly lower in Group 1 than in Group $2(p<0.0001)$ at $20(p<0.0001), 24(p<0.0001)$ and 26 weeks $(p<0.0001)$. Cumulative viral RNA loads in serum during days 1-10 post-challenge were significantly lower in Group 1 than in Group $2(p<0.0001)$ in all studies. A significant increase in ADWG was observed in Group 1 compared with Group 2 at 20 weeks $(p=0.0027)$ and 24 weeks $(p=0.0004)$, but not at 26 weeks $(p=0.1041)$. There were no significant differences in clinical signs post-challenge in any study.

Conclusion: These results suggest that Ingelvac PRRSFLEX ${ }^{\circledR}$ EU confers long-term immunity to European heterologous PRRSV, which is maintained up to 26 weeks after vaccination, corresponding to the expected lifespan of commercial pigs.

Keywords: Vaccine, Efficacy, PRRS, Immunity, Lung lesions, ADWG

\footnotetext{
* Correspondence: jeremy.kroll@boehringer-ingelheim.com

'Boehringer Ingelheim Animal Health, 2412 South Loop Dr, Ames, IA 50010,

USA

Full list of author information is available at the end of the article
} 


\section{Background}

Porcine reproductive and respiratory syndrome (PRRS) is a viral infection of pigs, causing pneumonia, reproductive failure, and increased mortality in young animals [1]. The disease was first identified in North America in the late 1980s [2] and soon after in Europe [3], and has since spread to other regions worldwide [4]. The disease has profound economic consequences, causing significant losses to the worldwide swine industry, and an estimated annual loss of 1 billion US dollars in North America alone [5]. The extent of animal suffering, along with these economic losses have led to a significant drive to develop effective prevention, control and elimination strategies.

PRRS is caused by the PRRS virus (PRRSV), which is a member of the Arteriviridae family of the order Nidovirales. Like all members of this family, PRRSV has a single-stranded, positive-sense RNA genome. There are two major genetic lineages: Type 1 , which was originally isolated in Europe in 1990 [6], and Type 2, which was first isolated in North America [7]. These two genotypes emerged at approximately the same time, but their nucleotide sequences differ by approximately $40 \%[8,9]$. Indeed, there is also significant genetic variation within each genotype $[5,10]$. This significant heterogeneity both between and within PRRSV genotypes has hindered attempts to develop an effective vaccine against PRRS.

At present, two types of vaccine are commercially available: killed-virus (KV) vaccines and modified-live virus (MLV) vaccines $[11,12]$. KV vaccines are largely ineffective, or have only limited efficacy [13-15] while MLV vaccines are generally regarded as the most effective [5], reducing reproductive and respiratory symptoms of PRRS disease, and improving weight gain in growing pigs [16].

Despite their promising efficacy, PRRS MLV vaccines are still associated with multiple problems. Commercially available PRRS MLV vaccines are based on either PRRSV Type 1 or 2 viral strains, which effectively induce immunity against genetically similar PRRSV strains from within the same genotype $[11,12]$. However, the level of efficacy conferred against heterologous PRRSV isolates is less clear. Vaccine efficacy can suffer from both the immune evasion strategies of the virus and the antigenic heterogeneity of the field strains. It is not possible to forecast precisely the level of protection afforded by a given PRRSV vaccine strain against a heterologous one, but it is clear that partial heterologous protection can be obtained [17-19].

Commercially-reared pigs are typically slaughtered between 18 and 26 weeks of age [20]; therefore, an optimal vaccine should quickly elicit an effective immune response, and maintain duration of immunity for at least 26 weeks. The absence of clear correlates of protection means that further in vivo studies are necessary [21]. Current PRRS MLV vaccines elicit humoral and cell- mediated immunity; both become detectable by $2-3$ weeks post-vaccination $[22,25]$. However, whilst levels of PRRSspecific antibodies rapidly reach their peak of detection 4 weeks after vaccination or infection, the cell-mediated response is profoundly delayed, remaining at low levels for over 3 months before reaching its highest levels after 32 weeks post-vaccination [22]. As well as their efficacy shortcomings, there also remain concerns over the safety of PRRS MLV vaccines due to their potential to revert to virulence [23, 24]. A vaccine conferring rapid, long-lasting as well as effective immunity against a broad range of heterologous viral strains is very much needed [11, 23].

An ideal vaccine would achieve a long duration of immunity to reduce the risk of re-infection with PRRS during an animal's lifetime. Simultaneously, it must effectively reduce clinical signs of PRRS disease, viraemia, viral RNA load in lung tissues and viral shedding, to help limit viral transmission. Such a vaccine would help limit both the acute economic losses caused by PRRS infection and animal suffering. Developing such a vaccine represents a significant challenge [23]. A new vaccine, PRRS 94881 (tradename: Ingelvac PRRSFLEX ${ }^{\circ}$ EU), is derived from European PRRS isolate 94881, and has previously been shown to be clinically safe [25]. In order to determine whether PRRS 94881 MLV could provide long-term immunity to PRRSV challenge in 2week old pigs, three vaccination-challenge studies following Good Clinical Practices (GCP) were carried out to evaluate the duration of immunity (DOI) at three different time points after vaccination. This paper details the results of these studies, which found that vaccinated pigs had reduced lung lesion scores, viraemia and viral RNA load in tissues at 20, 24 and 26 weeks.

\section{Methods \\ Study designs}

Three blinded, vaccination-challenge efficacy studies were performed using separate cohorts of pigs. Each study comprised three treatment arms. Group 1 (PRRSvaccinated) received Ingelvac PRRSFLEX ${ }^{\oplus}$ EU (Boehringer Ingelheim Vetmedica, Inc., St. Joseph, MO, USA; Lot 390-005) followed by PRRSV challenge. Group 2 (challenge controls) received control product (CP; Boehringer Ingelheim Vetmedica, Inc.; Lot N240-191-062409) followed by PRRSV challenge. Group 3 (negative controls) received $\mathrm{CP}$, but no PRRSV challenge. Ingelvac PRRSFLEX $^{\circledast}$ EU vaccine and CP were administered to 14-17 day-old pigs on Day 0, and then Group 1 and Group 2 were challenged with heterologous European PRRSV isolate 205,817 (Boehringer Ingelheim Vetmedica, Inc.) at 20,24 or 26 weeks after vaccination. This day was denoted day post-challenge (DPC) 0 . All studies were carried out following Good Clinical Practice (GCP) guidelines. 


\section{Randomisation and blinding}

Animals were randomised to one of three groups by the study investigator or a designee prior to study commencement. The randomisation sequence was created using Excel 2003 (Microsoft, Redmond, WA, US) with a 1:1 allocation using random block sizes of 0 and 1 . Both the Ingelvac PRRSFLEX ${ }^{\odot} \mathrm{EU}$ and $\mathrm{CP}$ were administered by individuals not collecting study data in order to maintain study blinding. Both the study investigator and all laboratory personnel were blinded to treatment assignment.

\section{Animals}

These three studies were each conducted with 56 commercial crossbred female or castrated male pigs (Prairie View Farms, N5627 Hwy DD, Burlington, WI 53105, USA). The animals were healthy, aged between 14 and 17 days, weighed between 2.7-6.3 kg and were PRRSVnegative on Day 0. All pigs were housed at Veterinary Resources, Inc. (VRI) in Cambridge, IA, USA, for the duration of the study. Pigs were housed in multiple pens (each containing 11-12 pigs) per room. Vaccinated (Group 1) and control (Groups 2 and 3) animals were housed in uniform but separate rooms to prevent PRRSV cross-contamination between groups. Feeds provided were appropriate for the size, age and condition of pigs according to acceptable animal husbandry practices for the region. A minimum of 20 pigs were included in Groups 1 and 2, and 12 pigs were included in Group 3.

\section{Vaccines and challenge material}

Both Ingelvac PRRSFLEX ${ }^{\oplus}$ EU vaccine and CP were reconstituted with phosphate-buffered saline. Ingelvac PRRSFLEX $^{\oplus}$ EU was reconstituted and administered to Group 1 animals per manufacturer's instructions [26]. Ingelvac PRRSFLEX ${ }^{\oplus}$ EU vaccine and $C P$ were administered intramuscularly (IM) as a $1.0 \mathrm{~mL}$ dose to the right neck region of Group 1 and 2 pigs, respectively. Challenge material was PRRSV isolate 205,817, a heterologous Type 1 isolate with $88.3 \%$ homology to Ingelvac PRRSFLEX ${ }^{\odot}$ EU (based on ORF5 sequence). The challenge strain was originally isolated from a herd with a severe outbreak of PRRS causing abortions in sows and respiratory disease in fattening pigs. Challenge material had a mean viral titre of $1 \times 10^{6.27} \mathrm{TCID}_{50} / 3 \mathrm{~mL}$ dose administered $1 \mathrm{~mL}$ per each nostril $(2 \mathrm{~mL}$ in total) and $1 \mathrm{~mL}$ intramuscularly. The CP was a lyophilised placebo product that contained an inert material comprising the vaccine vehicle without Ingelvac PRRSFLEX ${ }^{\odot}$ EU.

\section{Variables for efficacy and safety assessments}

The primary efficacy outcome variable for the three studies was lung pathology (gross and histological lesions) at 20, 24 or 26 weeks after PRRS 94881 MLV vaccination. The DOI at 20,24 and 26 weeks post- vaccination was achieved if Group 1 had statistically significant decreased lung pathology (gross or histological) post-challenge compared with Group 2 at the same time point. Secondary efficacy outcomes included post-challenge viraemia (in both lung and serum), average daily weight gain (ADWG), post-vaccination safety assessments and post-challenge clinical assessments of disease.

\section{Sample analysis and outcome measurements Gross and histological lung lesions}

Gross pathology was determined following examination of lung tissue following necropsy on DPC 10. A percentage of affected lung tissue was recorded for each lung lobe and the total percentage was subsequently calculated based on the weighing formula recommended in the draft monograph 'Porcine enzootic Pneumonia Vaccine (inactivated)' (PA/PH, Exp $15 \mathrm{~V} / \mathrm{T}[07] 2$ ANP). To determine histological pathology, a single slide containing seven sections (one each for all seven lung lobes) was created for each pig. Slides were examined for pneumocytic hypertrophy and hyperplasia, septal infiltration with mononuclear cells, necrotic debris, intra-alveolar accumulation of inflammatory cells, and perivascular accumulation of inflammatory cells. For each histological parameter (except necrotic debris), samples were scored either 0 (not present: no detectable lesions present within an area of view), 1 (mild lesions: few positive cells [1-5 cells/area] present within an area of view), 2 (moderate lesions: multiple positive cells [ $>5$ cells/area] within an area of view) or 3 (severe lesions: multiple positive cells [> 5 cells/ area] at multiple locations within an area of view). Necrotic debris was scored either 0 (not present) or 1 (yes present).

\section{Serum PRRSV quantitative polymerase chain reaction}

Two to $5 \mathrm{~mL}$ venous whole blood samples were collected from all pigs on Day 0 to confirm the PRRSV-negative status. Blood samples were also taken on DPC 0, 3, 7, 9 and 10 in all three studies. Reverse transcription and quantitative PCR (qPCR) were performed [27] on serum samples to determine serum PRRSV RNA levels; results were reported as genome equivalent/mL $\left(\log _{10} \mathrm{GE} / \mathrm{mL}\right)$.

\section{Lung PRRSV qPCR}

Tissue samples from left and right lung lobes were homogenised, and qPCR following reverse transcription was performed (BioScreen GmbH, Hannover, Germany) to determine lung PRRSV RNA levels [27]; results were reported as $\log _{10} \mathrm{GE} / \mathrm{mL}$.

\section{Average daily weight gain}

Body weights were recorded on Day 0, DPC 0 and DPC 9 in all studies, and individual daily weight gains were calculated between DPC 0-9. 


\section{Post-vaccination and post-challenge clinical safety observations}

Post-vaccination clinical safety assessments were performed daily on Days -1 to 21 , then three times per week thereafter until DPC - 2 in all studies. These clinical assessments were recorded as either 'normal' or 'abnormal'. Pigs were observed for clinical signs of disease from DPC -1 to 10 in all three studies. Clinical parameters included respiratory symptoms, behaviour and cough, and were scored $0-3$ based on severity of symptoms (Respiration: $0=$ normal respiration; 1 = panting/rapid respiration; 2 =dyspnoea; $3=$ dead. Behaviour: $0=$ normal; 1 = mild to moderate lethargy; 2 = severely lethargic or recumbent; 3 =dead. Cough: $0=$ no coughing; $1=$ soft or intermittent cough; 2 = harsh or severe, repetitive cough; 3 = dead).

\section{Statistical methods}

For each study, pigs were randomly assigned to one of three groups. Data were summarised using descriptive statistics with a 95\% confidence interval (CI), and analysed assuming a completely random design structure. All tests on differences between Groups 1 and 2 were designed as two-sided tests using an alpha value of 5\% ( $p$-value $<0.05$ for indicating statistical significance). Differences between treatment groups in each study were tested using analysis of variance in case of quantitative variables with normally distributed data (ADWG) and Wilcoxon Mann-Whitney tests for scores and other variables with not normally distributed data. Statistical analyses were performed using SAS software release 8.2 (SAS 2001, SAS Institute Inc., Cary, North Carolina, USA). No statistical analyses were performed on Group 3 pigs.

\section{Results}

\section{Animals}

In total, 56 animals were included per study, and assigned to one of three groups. For each study, Groups 1 and 2 comprised 22 animals each, and Group 3 comprised 12 animals. In the 20-week study, two pigs assigned to Group 2 died. In the 24-week study, one Group 1 pig and three Group 2 pigs died, and in the 26week study, one Group 1 pig and two Group 2 pigs died. All of these animals died pre-challenge, and were not included in the analyses presented here.

\section{Lung lesion scores}

The mean gross lung lesion scores for Group 1 pigs following challenges at 20, 24 and 26 weeks were $0.156 \%$, $0.084 \%$ and $1.099 \%$, respectively, whilst those for Group 2 pigs were $0.26 \%, 3.38 \%$ and $15.84 \%$, respectively (Table 1). The differences between the two groups were significant after challenges at 24 and 26 weeks, but not after 20 weeks (Table 1 ).
Table 1 Gross lung lesion scores at necropsy

\begin{tabular}{lllllll}
\hline & \multicolumn{6}{l}{ Gross lung lesion score (\%) } \\
\cline { 2 - 7 } Study & Group & $\mathrm{n}$ & Mean & $95 \% \mathrm{Cl}$ & Median (IQR) & $p$-value* \\
\hline 20-week & 1 & 22 & 0.156 & $0.00-0.16$ & $0.000(0.160)$ & 0.2989 \\
& 2 & 20 & 0.261 & $0.00-0.16$ & $0.060(0.185)$ & \\
24-week & 1 & 20 & 0.084 & $0.00-0.10$ & $0.000(0.130)$ & $<0.0001$ \\
& 2 & 19 & 3.378 & $0.61-4.74$ & $2.050(4.59)$ & \\
26-week & 1 & 21 & 1.099 & $0.05-0.55$ & $0.060(0.400)$ & $<0.0001$ \\
& 2 & 19 & 15.842 & $2.69-22.65$ & $13.80(20.85)$ & \\
\hline
\end{tabular}

$\mathrm{Cl}$ distribution free confidence interval of the median, IQR interquartile range, $M L V$ modified live virus, $n$ number of pigs included in analysis, PRRS porcine reproductive and respiratory syndrome

Group 1 = PRRS 94881 MLV vaccinated; Group 2 =challenge controls ${ }^{*} p$-values were calculated using the Wilcoxon-Mann-Whitney test

Group 1 pigs exhibited significantly lower mean histological lung lesion scores than Group 2 pigs after challenges at 20, 24 and 26 weeks (Table 2).

\section{Viral RNA load in serum}

PRRSV RNA was not detected in the serum of any pigs on Day 0, meeting the inclusion criteria for the study. Also, no PRRSV RNA was detected in any group on the day of challenge (DPC0), meaning that the vaccine virus was cleared at this point in time.

Group 1 pigs in all three studies exhibited significantly lower serum PRRSV RNA levels than Group 2 pigs at both DPC 7 and DPC 10 (Table 3). During DPC 1-10, the mean area under the curve (AUC) values for Group 1 pigs were $14.3,12.9$ and 17.6 $\log _{10} \mathrm{GE} / \mathrm{mL}$, and $37.4,35.3$ and $44.8 \log _{10} \mathrm{GE} / \mathrm{mL}$ for Group 2 pigs in the 20, 24 and 26-week studies, respectively (Table 4 ).

AUC values during DPC 3-10 were also significantly lower for Group 1 than Group 2 pigs in all three studies. Group 1 pigs exhibited mean AUC values of 8.57, 7.83 and $11.0 \log _{10} \mathrm{GE} / \mathrm{mL}$ compared with 29.4, 27.7 and 36 . $1 \log _{10} \mathrm{GE} / \mathrm{mL}$ for Group 2 pigs in the 20, 24 and 26week studies, respectively (Table 4).

Table 2 Mean histological lung lesion scores at necropsy

\begin{tabular}{lllllll}
\hline \multirow{5}{*}{ Study } & \multicolumn{6}{l}{ Histological lung lesion score (mean) } \\
\cline { 2 - 7 } & Group & $\mathrm{n}$ & Mean & $95 \% \mathrm{Cl}$ & Median (IQR) & $p_{\text {-value }}$ \\
\hline 20-week & 1 & 22 & 13.0 & $4.0-18.0$ & $9.5(14.0)$ & 0.0065 \\
& 2 & 20 & 24.7 & $16.0-35.0$ & $23.0(20.5)$ & \\
24-week & 1 & 20 & 7.3 & $2.0-10.0$ & $7.0(8.5)$ & $<0.0001$ \\
& 2 & 19 & 20.6 & $12.0-28.0$ & $19.0(16.0)$ & \\
26-week & 1 & 21 & 6.6 & $3.0-8.0$ & $6.0(5.0)$ & $<0.0001$ \\
& 2 & 20 & 20.2 & $15.0-23.0$ & $19.5(10.0)$ & \\
\hline
\end{tabular}

$\mathrm{Cl}$ distribution free confidence interval of the median, IQR interquartile range, $M L V$ modified live virus, $n$ number of pigs included in analysis, PRRS porcine reproductive and respiratory syndrome

Group 1 = PRRS 94881 MLV vaccinated; Group 2 = challenge controls ${ }^{*} p$-values were calculated using the Wilcoxon-Mann-Whitney test 
Table 3 PRRSV-viral RNA load in serum

\begin{tabular}{|c|c|c|c|c|c|c|c|}
\hline \multirow[b]{2}{*}{ Study } & \multirow[b]{2}{*}{ DPC } & \multicolumn{6}{|c|}{ PRSSV-viral RNA load in serum ( $\left.\log _{10} \mathrm{GE} / \mathrm{mL}\right)$} \\
\hline & & Group & $n$ & Mean & $95 \% \mathrm{Cl}$ & Median (IQR) & $p$-value ${ }^{*}$ \\
\hline \multirow[t]{8}{*}{ 20-week } & 0 & 1 & 22 & 0.00 & $0.00-0.00$ & $0.00(0.00)$ & 1.0000 \\
\hline & & 2 & 20 & 0.00 & $0.00-0.00$ & $0.00(0.00)$ & \\
\hline & 3 & 1 & 22 & 3.84 & $3.00-4.29$ & $3.79(1.29)$ & $<0.0001$ \\
\hline & & 2 & 20 & 5.30 & $5.06-5.52$ & $5.34(0.54)$ & \\
\hline & 7 & 1 & 22 & 0.14 & $0.00-0.00$ & $0.00(0.00)$ & $<0.0001$ \\
\hline & & 2 & 20 & 4.00 & 3.44-4.59 & $3.95(1.26)$ & \\
\hline & 10 & 1 & 22 & 0.27 & $0.00-0.00$ & $0.00(0.00)$ & $<0.0001$ \\
\hline & & 2 & 20 & 3.23 & $3.00-3.37$ & $3.00(0.51)$ & \\
\hline \multirow[t]{8}{*}{ 24-week } & 0 & 1 & 20 & 0.00 & $0.00-0.00$ & $0.00(0.00)$ & 1.0000 \\
\hline & & 2 & 19 & 0.00 & $0.00-0.00$ & $0.00(0.00)$ & \\
\hline & 3 & 1 & 20 & 3.39 & $3.00-4.04$ & $3.78(1.13)$ & $<0.0001$ \\
\hline & & 2 & 19 & 5.06 & $4.85-5.36$ & $5.13(0.56)$ & \\
\hline & 7 & 1 & 19 & 0.32 & $0.00-0.00$ & $0.00(0.00)$ & $<0.0001$ \\
\hline & & 2 & 19 & 3.85 & $3.00-4.53$ & $3.87(1.53)$ & \\
\hline & 10 & 1 & 20 & 0.00 & $0.00-0.00$ & $0.00(0.00)$ & $<0.0001$ \\
\hline & & 2 & 19 & 2.75 & $3.00-3.37$ & $3.00(0.37)$ & \\
\hline \multirow[t]{8}{*}{ 26-week } & 0 & 1 & 21 & 0.00 & $0.00-0.00$ & $0.00(0.00)$ & 1.0000 \\
\hline & & 2 & 20 & 0.00 & $0.00-0.00$ & $0.00(0.00)$ & \\
\hline & 3 & 1 & 21 & 4.42 & $3.93-5.28$ & $4.44(1.51)$ & $<0.0001$ \\
\hline & & 2 & 20 & 5.81 & $5.75-6.00$ & $5.88(0.32)$ & \\
\hline & 7 & 1 & 21 & 0.61 & $0.00-0.00$ & $0.00(0.00)$ & $<0.0001$ \\
\hline & & 2 & 20 & 5.30 & $4.86-5.69$ & $5.30(1.08)$ & \\
\hline & 10 & 1 & 21 & 0.00 & $0.00-0.00$ & $0.00(0.00)$ & $<0.0001$ \\
\hline & & 2 & 20 & 3.97 & $3.71-4.42$ & $4.24(1.18)$ & \\
\hline
\end{tabular}

$\mathrm{Cl}$ distribution free confidence interval of the median, DPC day post-challenge, GE genome equivalent, $I Q R$ interquartile range, $M L V$ modified live virus, $n$ number of pigs included in analysis, PRRS porcine reproductive and respiratory syndrome, PRRSV PRRS virus

Group 1 = PRRS 94881 MLV vaccinated; Group 2 = challenge controls

* $p$-values were calculated using the Wilcoxon-Mann-Whitney test

\section{Viral RNA load in lung tissues at necropsy}

Lung PRRSV RNA levels at necropsy for Group 1 pigs were significantly lower than for Group 2 pigs in all three studies $(p<0.0001$; Table 5).

\section{Average daily weight gain}

At the time of challenge, the mean weight difference between respective groups was insignificant. A significant increase in ADWG was observed in Group 1 pigs compared with Group 2 pigs in the 9 days following challenge at $20(p=0.0027)$ and $24 \quad(p=0$. 0004) weeks, but not at 26 weeks $(p=0.1041)$ (Table 6). Mean ADWG in Group 3 pigs was 1.016, 0.771 and $0.5 \mathrm{~kg} /$ day in the 9 days following challenge at 20, 24 and 26 weeks, respectively. This was greater than the ADWG of both Group 1 and 2 pigs in all three studies.

\section{Clinical signs post-challenge}

For all three studies, no difference was observed between Group 1 and Group 2 for each of the clinical signs considered: abnormal respiration, abnormal behaviour and coughing. No pigs in either Group 1 or Group 2 exhibited any coughing following challenge at 20, 24 or 26 weeks. No abnormal respiration, behaviour or coughing was observed in any pigs challenged at 20 weeks.

\section{Adverse events}

No adverse events attributed to the test product (Ingelvac PRRSFLEX $\left.^{\odot} \mathrm{EU}\right)$ was noted in any of the three studies. Also, no deaths were attributed to either control product or Ingelvac PRRSFLEX EU.

Table 4 PRRSV-viral RNA cumulative loads in serum

\begin{tabular}{|c|c|c|c|c|c|c|c|}
\hline \multirow[b]{2}{*}{ Study } & \multirow[b]{2}{*}{ DPC } & \multirow[b]{2}{*}{ Group } & \multicolumn{5}{|c|}{ AUC values ( $\left.\log _{10} \mathrm{GE} / \mathrm{mL}\right)$} \\
\hline & & & $\bar{n}$ & Mean & $95 \% \mathrm{Cl}$ & Median (IQR) & $p$-value ${ }^{*}$ \\
\hline \multirow[t]{4}{*}{ 20-week } & $0-10$ & 1 & 22 & 14.33 & $10.50-17.80$ & $13.32(7.3)$ & $<0.0001$ \\
\hline & & 2 & 20 & 37.40 & $35.02-39.37$ & $37.44(4.54)$ & \\
\hline & $3-10$ & 1 & 22 & 8.57 & $6.00-10.46$ & $7.61(4.46)$ & $<0.0001$ \\
\hline & & 2 & 20 & 29.44 & $27.71-31.08$ & $29.74(3.76)$ & \\
\hline \multirow[t]{4}{*}{ 24-week } & $0-10$ & 1 & 19 & 12.87 & $10.50-15.16$ & $13.23(4.66)$ & $<0.0001$ \\
\hline & & 2 & 19 & 35.31 & $32.78-38.22$ & 36.07 (5.68) & \\
\hline & $3-10$ & 1 & 19 & 7.83 & $6.00-8.86$ & $7.56(2.86)$ & $<0.0001$ \\
\hline & & 2 & 19 & 27.72 & $25.16-30.72$ & $28.29(5.70)$ & \\
\hline \multirow[t]{4}{*}{ 26-week } & $0-10$ & 1 & 21 & 17.61 & $13.76-19.53$ & $15.54(5.95)$ & $<0.0001$ \\
\hline & & 2 & 20 & 44.84 & $43.23-48.03$ & $44.77(6.24)$ & \\
\hline & $3-10$ & 1 & 21 & 10.97 & $7.86-11.16$ & $8.88(3.40)$ & $<0.0001$ \\
\hline & & 2 & 20 & 36.12 & $34.60-38.53$ & 36.43 (5.23) & \\
\hline
\end{tabular}

$A U C$ area under the concentration-time curve, $C l$ distribution free confidence interval of the median, $D P C$ day post-challenge, GE genome equivalent, $I Q R$ interquartile range, $M L V$ modified live virus, $n$ number of pigs included in analysis, PRRS porcine reproductive and respiratory syndrome, $P R R S V$ PRRS virus Group 1 = PRRS 94881 MLV vaccinated; Group 2 = challenge controls * $p$-values were calculated using the Wilcoxon-Mann-Whitney test 
Table 5 PRRSV-viral RNA load for lung tissues at necropsy

\begin{tabular}{|c|c|c|c|c|c|c|}
\hline \multirow[b]{2}{*}{ Study } & \multicolumn{6}{|c|}{ PRRSV-viral RNA load in lung tissues at necropsy (mean $\log _{10} \mathrm{GE} / \mathrm{mL}$ ) } \\
\hline & Group & $\mathrm{n}$ & Mean & $95 \% \mathrm{Cl}$ & Median (IQR) & $p$-value \\
\hline \multirow[t]{2}{*}{ 20-week } & 1 & 22 & 4.26 & $3.70-5.18$ & $4.20(1.51)$ & $<0.0001$ \\
\hline & 2 & 20 & 6.31 & $5.94-6.73$ & $6.51(0.86)$ & \\
\hline \multirow[t]{2}{*}{ 24-week } & 1 & 20 & 2.36 & $1.50-4.17$ & $1.50(3.42)$ & $<0.0001$ \\
\hline & 2 & 19 & 5.33 & $4.85-5.97$ & $5.34(1.23)$ & \\
\hline \multirow[t]{2}{*}{ 26-week } & 1 & 21 & 3.36 & $1.50-5.21$ & 3.69 (3.18) & $<0.0001$ \\
\hline & 2 & 20 & 6.22 & $5.62-6.68$ & $6.25(1.26)$ & \\
\hline
\end{tabular}

$\mathrm{Cl}$ distribution free confidence interval of the median, GE genome equivalent, $I Q R$ interquartile range, $M L V$ modified live virus, $n$ number of pigs included in analysis, PRRS porcine reproductive and respiratory syndrome, PRRSV PRRS virus

Group 1 = PRRS $94881 \mathrm{MLV}$ vaccinated; Group 2 = challenge controls

" $p$-values were calculated using the Wilcoxon-Mann-Whitney test

\section{Discussion}

These three GCP laboratory efficacy studies were performed in seronegative 2-week old pigs. Each study contained three groups; Group 1 was vaccinated on Day 0 with Ingelvac PRRSFLEX EU, and Groups 2 and 3 were vaccinated with a placebo vaccine. This study aimed to evaluate DOI at 20,24 and 26 weeks following vaccination. After 20, 24 or 26 weeks, Groups 1 and 2 were challenged with a heterologous European PRRS viral isolate 205,817 , which shared $88.3 \%$ sequence homology at the GP5 gene with Ingelvac PRRSFLEX ${ }^{\oplus}$ EU. The GP5 gene encodes the major envelope protein of PRRSV, and carries the major neutralising epitope of PRRSV [28]. Groups 1 and 2 were subsequently evaluated for lung pathology, serum and lung viremia, and observed for clinical signs of disease. No infections were observed in Group 3 pigs in any study, and there were no signs of co-infection. Therefore, the results presented here were attributed to the effects of PRRS 94881 MLV vaccine.

PRRS respiratory infection models using EU type 1 PRRSV are rare due to them being difficult to develop. In the current study, we have modelled a novel PRRSV type I isolate 205,817 that can consistently infect and cause severe PRRS respiratory lesions at different ages of a growing and fattening pig; however, the amount of

Table 6 Mean group ADWG during DPC 0-9

\begin{tabular}{|c|c|c|c|c|c|}
\hline \multicolumn{6}{|c|}{ Mean ADWG (kg/day) } \\
\hline Study & Group & $\mathrm{n}$ & Mean (SD) & Median & $p$-value \\
\hline \multirow[t]{2}{*}{ 20-week } & 1 & 22 & $0.740(0.333)$ & 0.739 & 0.0027 \\
\hline & 2 & 20 & $0.155(0.783)$ & 0.339 & \\
\hline \multirow[t]{2}{*}{ 24-week } & 1 & 20 & $0.737(0.295)$ & 0.683 & 0.0004 \\
\hline & 2 & 19 & $0.068(0.705)$ & 0.189 & \\
\hline \multirow[t]{2}{*}{ 26-week } & 1 & 21 & $0.412(0.317)$ & 0.411 & 0.1041 \\
\hline & 2 & 20 & $0.235(0.365)$ & 0.289 & \\
\hline
\end{tabular}

ADWG average daily weight gain, $M L V$ modified live virus, $n$ number of pigs included in analysis, PRRS porcine reproductive and respiratory syndrome, $S D$ standard deviation

Group 1 = PRRS 94881 MLV vaccinated; Group 2 = challenge controls

* $p$-values were calculated using the analysis of variance (t-test) expected clinical signs and respiratory disease is reduced the older the pig gets.

Reduction in gross and histological lung lesion scores was the primary efficacy endpoint of three studies. Lung lesion development is one of the hallmarks of PRRSV in growing pigs [29], and can be considered the source for all subsequent manifestations of secondary PRRS disease characteristics, including clinical signs, pyrexia, decreased ADWG and secondary infection with other pathogens. Therefore, these are the most clinically relevant and convincing parameters for measurement of PRRS vaccine efficacy. Macroscopic gross lung lesions are usually mild in adult animals, so slides were examined for multiple microscopic lung lesions typically observed following PRRSV infection [30]. The results of these three studies showed significant improvements in both gross and histological lung lesion scores among Group 1 compared with Group 2 pigs at 24 and 26 weeks after vaccination. Additionally, a significant reduction in histological lung lesions was seen at 20 weeks in Group 1 pigs. In the 20-week study, the lung lesion score of Group 1 animals was in a similar range to that of Group 1 animals in the two adjacent studies; however, significance could not be reached due to the fact that lung lesions in Group 2 pigs were low as well. These findings suggest that Ingelvac PRRSFLEX ${ }^{\circ}$ EU vaccine is highly effective in providing long-term (up to 26 weeks) immunity against a virulent challenge with PRRS. Some histological lung lesions were observed in negative control pigs, despite their confirmed absence of PRRSV RNA in serum at all time points throughout the study. Pigs housed under normal swine husbandry conditions for extended periods of time can develop minor lung lesions that are inconsequential, and not related to specific pathogens [31].

Post-challenge viremia was selected as the most important secondary efficacy parameter because it represents the level of viral replication occurring within the host animal upon exposure. Furthermore, pathogenic and humoral immune responses to PRRSV are related to 
viral loads in acute infection [32]. Therefore, a significant reduction in PRRS viral load following vaccination would indicate that the vaccine could efficiently limit PRRSV pathogenesis in the host. Significant reductions in post-challenge viremia were observed in Group 1 pigs compared with Group 2 pigs 7 and 10 days postchallenge at 20-26 weeks. Cumulative exposure (AUC) from Days 3-10 post-challenge was also decreased in these pigs. Both findings indicate that Ingelvac PRRSFLEX $^{\circ}$ EU is efficient in limiting PRRSV viremia following virulent challenge, and confers a duration of immunity up to 26 weeks. Furthermore, a significant reduction in PRRSV-viral RNA load in lung tissue was observed in Group 1 pigs compared with Group 2 pigs at 20,24 and 26 weeks. Viral RNA load in lung tissue is associated with viral replication and persistence in the host [33]. The results of these studies showed that Ingelvac PRRSFLEX ${ }^{ø}$ EU can effectively reduce PRRS-viral RNA load in lung tissue.

PRRSV infection contributes to a reduction in daily weight gain in young pigs [34]. In the 9 days following PRRSV challenge at 20 and 24 weeks, the ADWG of the Group 1 pigs was significantly higher than that of Group 2 pigs, whose ADWG was lower than that of Group 3 pigs. Following challenge at 26 weeks, ADWG in Group 1 pigs was increased compared with Group 2, but this increase did not reach statistical significance. These results indicate that Ingelvac PRRSFLEX ${ }^{\circ}$ EU is effective at diminishing weight gain reduction caused by PRRS, possibly representing substantial economic importance. A recently published field study also supports this conclusion. This study found that vaccinating 4-week old piglets with Ingelvac PRRSFLEX ${ }^{\circ}$ EU improved weight gain and reduced clinical signs during the growing period, even when the piglets are infected shortly after vaccination [35]. However, the current study is limited by the lack of statistical power due to the small sample size and findings need to be confirmed in larger studies. Also, this study used young pigs that were negative for PRRSV RNA; further studies are therefore required to determine whether these results apply to pigs of different ages and immune status.

PRRS MLV vaccines are considered the most effective PRRS vaccines available $[5,12]$. The results of the previous studies provide evidence that Ingelvac PRRSFLEX ${ }^{\circ}$ EU effectively confers immunity to PRRSV, which is maintained for up to 26 weeks. Since commercially-reared animals are typically slaughtered between 18 and 26 weeks of age [20], this long DOI conferred by Ingelvac PRRSFLEX ${ }^{\circ}$ EU may help reduce the chance of PRRSV-related disease for the whole lifespan of vaccinated animals.

\section{Conclusions}

The three studies described here show that Ingelvac PRRSFLEX ${ }^{\circ}$ EU was highly effective at conferring long- term immunity (up to 26 weeks) against virulent challenge with a heterologous European isolate of PRRSV in young pigs. After challenge at 24 and 26 weeks, gross and histological lung lesion scores, post-challenge viremia and viral RNA load in lung tissue, and ADWG were improved in PRRS-vaccinated pigs compared with challenge controls, with most measures reaching statistical significance. These results show that protection against heterologous PRRSV is achieved with the Ingelvac PRRSFLEX $\mathrm{X}^{\circ} \mathrm{EU}$, and can be maintained for the expected lifespan of young pigs, with beneficial effects on animal health and production.

\section{Abbreviations}

ADWG: average daily weight gain; AUC: area under the concentration-time curve; Cl: confidence interval; CP: control product; DOl: duration of immunity; DPC: day post-challenge; GCP: good clinical practices; GE: genome equivalent; IM: intramuscular; IQR: interquartile range; KV: killed virus; MLV: modified live virus; PRRS: porcine reproductive and respiratory syndrome; PRRSV: porcine reproductive and respiratory syndrome virus; QPCR: quantitative polymerase chain reaction; TCID: tissue culture infective dose; VRl: Veterinary Resources, Inc.

\section{Acknowledgements}

The authors thank Dr. Ryan Saltzman, Dr. Lyle Keel, Dr. Stephan Perch, Mr. Rex Smiley, Dr. Alicia Zimmerman and Ms. Sarah Layton for technical assistance. The authors would also like to thank Dr. Zoe Kelly of InterComm International Ltd., Cambridge, UK, for providing medical writing support in accordance with Good Publication Practice (GPP3) guidelines (http:// www.ismpp.org/gpp3).

\section{Funding}

The three studies were funded by Boehringer Ingelheim Vetmedica, Inc. Medical writing support with this manuscript was provided by InterComm International, Cambridge, UK, and this service was funded by Boehringer Ingelheim Vetmedica, Inc.

\section{Availability of data and materials}

The datasets generated and/or analysed during the current study are available in the BIAH Biological R\&D repository. The original datasets used and/or analysed during the current study are the private property of BIAH. All data generated or analysed during this study are included in this published article and its supplementary information files.

\section{Authors' contributions}

JK, F-XO, CK and MP designed the study outlines, monitored the studies, reviewed, analysed and reported the data. PR and OD reviewed the manuscript. All authors read and approved the final manuscript.

\section{Ethics approval}

This study was approved by Boehringer Ingelheim Animal Health (BIAH) animal welfare committee. The animals were purchased by and remained in ownership of BIAH throughout the study.

\section{Consent for publication}

Not applicable.

\section{Competing interests}

Jeremy Kroll is an employee of Boehringer Ingelheim Vetmedica, Inc. Poul H Rathkjen, Francois-Xavier Orveillon, and Oliver G. Duran are employees of Boehringer Ingelheim Vetmedica GmbH, Christian Kraft is an employee of Boehringer Ingelheim Veterinary Research Center GmbH \& Co. KG. Michael Piontkowski is a retired employee of Boehringer Ingelheim Vetmedica, Inc.

\section{Publisher's Note}

Springer Nature remains neutral with regard to jurisdictional claims in published maps and institutional affiliations. 


\section{Author details}

'Boehringer Ingelheim Animal Health, 2412 South Loop Dr, Ames, IA 50010, USA. ${ }^{2}$ Boehringer Ingelheim Animal Health, 2621 North Belt Highway, St. Joseph, MO 64506, USA. ${ }^{3}$ Boehringer Ingelheim Vetmedica GmbH, Binger Straße 173, 55216 Ingelheim, Germany. ${ }^{4}$ Boehringer Ingelheim Veterinary Research Center GmbH \& Co. KG, Bemeroder Str. 31, 30559 Hannover, Germany.

\section{Received: 30 November 2017 Accepted: 26 March 2018}

Published online: 16 May 2018

\section{References}

1. Lunney JK, Benfield DA, Rowland RR. Porcine reproductive and respiratory syndrome virus: an update on an emerging and re-emerging viral disease of swine. Virus Res. 2010;154(1-2):1-6. Epub 19 Oct 2010

2. Keffaber KK. Reproductive failure of unknown etiology. Am Assoc Swine Pract Newsl. 1989;1:1-9.

3. Office International des Epizooties: World Animal Health 1991. Animal health status and disease control methods (part one: reports). 1992. http:// www.oie.int/doc/ged/D7827.PDF Accessed 10 Sept 2017.

4. Wills RW, Zimmerman JJ, Yoon KJ, Swenson SL, McGinley MJ, Hill HT, et al. Porcine reproductive and respiratory syndrome virus: a persistent infection. Vet Microbiol. 1997:55(1-4):231-40. Epub 01 Apr 1997

5. Renukaradhya GJ, Meng XJ, Calvert JG, Roof M, Lager KM. Live porcine reproductive and respiratory syndrome virus vaccines: current status and future direction. Vaccine. 2015:33(33):4069-80. Epub 08 July 2015

6. Terpstra C, Wensvoort G, Pol JM. Experimental reproduction of porcine epidemic abortion and respiratory syndrome (mystery swine disease) by infection with Lelystad virus: Koch's postulates fulfilled. Vet Q. 1991;13(3): 131-6. Epub 01 July 1991

7. Benfield DA, Nelson E, Collins JE, Harris L, Goyal SM, Robison D, et al. Characterization of swine infertility and respiratory syndrome (SIRS) virus (isolate ATCC VR-2332). J Vet Diagn Invest. 1992;4(2):127-33. Epub 01 Apr 1992

8. Nelsen CJ, Murtaugh MP, Faaberg KS. Porcine reproductive and respiratory syndrome virus comparison: divergent evolution on two continents. J Virol. 1999;73(1):270-80

9. $\quad$ PGW P. Porcine reproductive and respiratory syndrome virus: origin hypothesis. Emerg Infect Dis. 2003;9(8):903-8.

10. Kim WI, Lee DS, Johnson W, Roof M, Cha SH, Yoon KJ. Effect of genotypic and biotypic differences among PRRS viruses on the serologic assessment of pigs for virus infection. Vet Microbiol. 2007:123(1-3):1-14. Epub 01 May 2007

11. Charerntantanakul W. Porcine reproductive and respiratory syndrome virus vaccines: immunogenicity, efficacy and safety aspects. World J Virol. 2012; 1(1):23-30. Epub 12 Feb 2012

12. Rowland RR, Lunney J, Dekkers J. Control of porcine reproductive and respiratory syndrome (PRRS) through genetic improvements in disease resistance and tolerance. Front Genet. 2012;3:260. Epub 14 Feb 2013

13. Nielsen $T L$, Nielsen J, Have P, Baekbo P, Hoff-Jorgensen R, Botner A Examination of virus shedding in semen from vaccinated and from previously infected boars after experimental challenge with porcine reproductive and respiratory syndrome virus. Vet Microbiol. 1997:54(2):10112. Epub 01 Feb 1997

14. Nilubol D, Platt KB, Halbur PG, Torremorell M, Harris DL. The effect of a killed porcine reproductive and respiratory syndrome virus (PRRSV) vaccine treatment on virus shedding in previously PRRSV infected pigs. Vet Microbiol. 2004;102(1-2):11-8. Epub 04 Aug 2004

15. Scortti M, Prieto C, Alvarez E, Simarro I, Castro JM. Failure of an inactivated vaccine against porcine reproductive and respiratory syndrome to protect gilts against a heterologous challenge with PRRSV. Vet Rec. 2007;161(24): 809-13. Epub 18 Dec 2007

16. Cano JP, Dee SA, Murtaugh MP, Pijoan C. Impact of a modified-live porcine reproductive and respiratory syndrome virus vaccine intervention on a population of pigs infected with a heterologous isolate. Vaccine. 2007; 25(22):4382-91. Epub 25 Apr 2007

17. Park C, Choi $\mathrm{K}$, Jeong J, Chae C. Cross-protection of a new type 2 porcine reproductive and respiratory syndrome virus (PRRSV) modified live vaccine (Fostera PRRS) against heterologous type 1 PRRSV challenge in growing pigs. Vet Microbiol. 2015;177(1-2):87-94. Epub 15 Mar 2015

18. Prieto C, Alvarez E, Martinez-Lobo FJ, Simarro I, Castro JM. Similarity of European porcine reproductive and respiratory syndrome virus strains to vaccine strain is not necessarily predictive of the degree of protective immunity conferred. Vet J. 2008;175(3):356-63. Epub 15 June 2007

19. Roca M, Gimeno M, Bruguera S, Segales J, Diaz I, Galindo-Cardiel IJ, et al. Effects of challenge with a virulent genotype II strain of porcine reproductive and respiratory syndrome virus on piglets vaccinated with an attenuated genotype I strain vaccine. Vet J. 2012;193(1):92-6. Epub 24 Oct 2012

20. Marchant-Forde JN. Introduction to the welfare of pigs. In: Marchant-Forde JN, editor. The welfare of pigs. Dordrecht: Springer Netherlands; 2009. p. 1-12.

21. Murtaugh MP, Genzow M. Immunological solutions for treatment and prevention of porcine reproductive and respiratory syndrome (PRRS). Vaccine. 2011:29(46):8192-204. Epub 20 Sept 2011

22. Meier WA, Galeota J, Osorio FA, Husmann RJ, Schnitzlein WM, Zuckermann FA. Gradual development of the interferon-gamma response of swine to porcine reproductive and respiratory syndrome virus infection or vaccination. Virology. 2003;309(1):18-31. Epub 03 May 2003

23. $\mathrm{Hu}$ J, Zhang C. Porcine reproductive and respiratory syndrome virus vaccines: current status and strategies to a universal vaccine. Transbound Emerg Dis. 2014;61(2):109-20.

24. Madsen KG, Hansen CM, Madsen ES, Strandbygaard B, Botner A, Sorensen $\mathrm{KJ}$. Sequence analysis of porcine reproductive and respiratory syndrome virus of the American type collected from Danish swine herds. Arch Virol. 1998:143(9):1683-700. Epub 27 Oct 1998

25. Piontkowski M, Kroll J, Kraft C, Coll T. Safety and early onset of immunity with a novel European porcine reproductive and respiratory syndrome virus vaccine in young piglets. Can J Vet Res. 2016;80(2):124-33.

26. Summary of product characteristics. Ingelvac PRRSFLEX EU lyophilisate and solvent for suspension for injection for pigs. 2015: Health Products Regulatory Authority; 2017. http://www.hpra.ie/img/uploaded/swedocuments/ LicenseSPC_10007-052-001_02042015120937.pdf Accessed 10 Sept 2017

27. Revilla-Fernandez S, Wallner B, Truschner K, Benczak A, Brem G, Schmoll F, et al. The use of endogenous and exogenous reference RNAs for qualitative and quantitative detection of PRRSV in porcine semen. J Virol Methods. 2005:126(1-2):21-30. Epub 26 Apr 2005

28. Roques E, Girard A, St-Louis M-C, Massie B, Gagnon CA, Lessard M, et al. Immunogenic and protective properties of GP5 and M structural proteins of porcine reproductive and respiratory syndrome virus expressed from replicating but nondisseminating adenovectors. Vet Res. 2013:44(1):17.

29. Christianson WT, Joo HS. Porcine reproductive and respiratory syndrome: a review. J Swine Health Prod. 1994;2(2):10-28.

30. Rossow KD. Porcine reproductive and respiratory syndrome. Vet Pathol. 1998;35(1):1-20. Epub 17 Apr 1998

31. Grest P, Keller H, Sydler T, Pospischil A. The prevalence of lung lesions in pigs at slaughter in Switzerland. Schweizer Archiv fur Tierheilkunde. 1997; 139(11):500-6. Epub 01 Jan 1997

32. Johnson W, Roof M, Vaughn E, Christopher-Hennings J, Johnson CR, Murtaugh MP. Pathogenic and humoral immune responses to porcine reproductive and respiratory syndrome virus (PRRSV) are related to viral load in acute infection. Vet Immunol Immunopathol. 2004;102(3):233-47. Epub 28 Oct 2004

33. Rossow KD, Collins JE, Goyal SM, Nelson EA, Christopher-Hennings J, Benfield DA. Pathogenesis of porcine reproductive and respiratory syndrome virus infection in gnotobiotic pigs. Vet Pathol. 1995;32(4):361-73. Epub 1995/07/01

34. Li MM, Seelenbinder KM, Ponder MA, Deng L, Rhoads RP, Pelzer KD, et al. Effects of porcine reproductive and respiratory syndrome virus on pig growth, diet utilization efficiency, and gas release from stored manure. J Anim Sci. 2015;93(9):4424-35. Epub 07 Oct 2015

35. Cano G, Cavalcanti MO, Orveillon F-X, Kroll J, Gomez-Duran O, Morillo A, et al. Production results from piglets vaccinated in a field study in Spain with a type 1 porcine respiratory and reproductive virus modified live vaccine. Porcine Health Management. 2016:2(1):22. 